\title{
Approximations of Discrete, Continuous, and Hybrid Systems
}

\author{
George J. Pappas \\ School of Engineering and Applied Science \\ University of Pennsylvania \\ Philadelphia, U.S.A \\ http://www.seas.upenn.edu/ pappasg/
}

\begin{abstract}
Concurrency theory and formal verification of discrete systems have resulted in a wealth of system relationships, including the notions of language inclusion, simulation and bisimulation relations. These notions have had great impact in not only reducing the analysis complexity of discrete systems, but also in reducing problems for continuous and hybrid systems to purely discrete problems.

Such relationships for discrete systems require system observations to be identical. When interacting with the physical world, modeled by continuous or hybrid systems, exact relationships are restrictive and not robust. In this talk, we will present a framework of system approximation that applies to discrete, continuous, and hybrid systems by developing notions of approximate language inclusion, approximate simulation, and approximate bisimulation relations. We define a hierarchy of approximation pseudo-metrics between two systems that quantify the quality of the approximation, and capture the established exact relationships as zero sections.
\end{abstract}

Algorithms are developed for computing the proposed pseudo-metrics, both exactly and approximately. The exact algorithms require the generalization of the fixed point algorithms for computing simulation and bisimulation relations, or dually, the solution of a static game whose cost is the so-called branching distance between the systems. Approximations for the pseudo-metrics can be obtained by considering Lyapunov-like functions called simulation and bisimulation functions. We will present algorithms for computing the proposed pseudo-metrics for a variety of classes of continuous, hybrid systems, and stochastic hybrid systems, as well as in approximating continuous systems by purely discrete ones. 